\title{
Competition between Ta-Ta and Te-Te bonding leading to the commensurate charge density wave in $\mathrm{TaTe}_{4}$
}

\author{
Bogdan Guster \\ Catalan Institute of Nanoscience and Nanotechnology (ICN2), \\ CSIC and The Barcelona Institute of Science and Technology, Campus Bellaterra, 08193 Barcelona, Spain \\ and European Theoretical Spectroscopy Facility, Institute of Condensed Matter and Nanosciences, Université catholique de Louvain, \\ Chemin des étoiles 8, bte L07.03.01, B-1348 Louvain-la-Neuve, Belgium \\ Miguel Pruneda and Pablo Ordejón ๔ \\ Catalan Institute of Nanoscience and Nanotechnology (ICN2), \\ CSIC and The Barcelona Institute of Science and Technology, Campus Bellaterra, 08193 Barcelona, Spain \\ Enric Canadell $\odot$ \\ Institut de Ciència de Materials de Barcelona (ICMAB-CSIC), Campus UAB, 08193 Bellaterra, Spain
}

(Received 30 November 2021; accepted 8 February 2022; published 22 February 2022)

\begin{abstract}
The origin of the charge density wave in $\mathrm{TaTe}_{4}$ is discussed on the basis of a first-principles density functional theory analysis of the Fermi surface, electron-hole response function, phonon band structure of the average structure, and structural optimization of the modulated phase. Analysis of the band structure and Fermi surface of the average structure clearly proves that despite the presence of $\mathrm{TaTe}_{4}$ chains in the crystal structure, $\mathrm{TaTe}_{4}$ is in fact a 3D material as far as the electronic structure near the Fermi level is concerned. A Fermi surface nesting mechanism is dismissed as the origin of the $2 a \times 2 a \times 3 c$ structural modulation. The optimized $2 a \times 2 a \times 3 c$ structure, which is found to be the more stable modulation in agreement with the experimental observations, can be obtained directly from a soft-phonon mode computed for the undistorted structure. Our results suggest that the driving force for the distortion is the maximization of Ta-Ta metal-metal bonding subject to inducing the minimum bonding decrease in the Te sublattice.
\end{abstract}

DOI: 10.1103/PhysRevB.105.064107

\section{INTRODUCTION}

The transition metal tetratellurides $\mathrm{NbTe}_{4}$ [1] and $\mathrm{TaTe}_{4}$ $[2,3]$ have recently been the object of considerable attention in the context of the search for materials with high magnetoresistance and the competition between charge density waves (CDWs) and superconductivity [4-7]. Transition metal tellurides often exhibit crystal structures and transport properties differing from those of the corresponding selenides or sulphides [8]. The tellurium $5 p$ orbitals are considerably more diffuse than those of sulfur $3 p$ and selenium $4 p$, so the valence bands in transition metal tellurides are wider than those of selenides or sulphides and may overlap substantially with the bottom part of the transition-metal-based $d$ bands. This leads to a non-negligible electron transfer from tellurium to the transition metal [9] and, as a consequence, the formal $d$-electron count for the transition metal atom is often not obvious. These electron transfers have strong implications for the structural and transport properties of many transition metal tellurides.

Group V tetratelurides provide a clear example of such a situation. $\mathrm{NbTe}_{4}$ and $\mathrm{TaTe}_{4}$ are described as containing onedimensional (1D) chains of transition metal atoms which are coordinated by the tellurium atoms in a square antiprismatic way (Fig. 1). $\mathrm{VS}_{4}$ exhibits a related crystal structure built from $\mathrm{VS}_{4}$ rectangular antiprismatic chains $[10,11]$ and has the same number of valence electrons. $\mathrm{VS}_{4}$ is a semiconductor as a result of the usual Peierls dimerization of a chain of transition metal atoms with a $d^{1}$-electron count (i.e., possessing a half-filled quasi-1D band). In contrast, $\mathrm{NbTe}_{4}$ and $\mathrm{TaTe}_{4}$ are metallic, their high temperature crystal structure contains uniform chains, and exhibit structural modulations toward a $2 a \times 2 a \times 3 c$ commensurate phase. $\mathrm{TaTe}_{4}$ has a transition to this commensurate phase at around $450 \mathrm{~K}$ [12] while its isostructural counterpart, $\mathrm{NbTe}_{4}$, crosses several incommensurate phases before locking into the final commensurate one at $50 \mathrm{~K}[13,14]$. Because of the presence of chains of transition metal atoms and the high-temperature metallic character, these CDWs have been attributed to a Peierls-type mechanism as in other quasi-1D metals [15-17]. However, in contrast with other quasi-1D chalcogenides exhibiting structural modulations like $\mathrm{NbSe}_{3}$ or monoclinic $\mathrm{TaS}_{3}$ [8], the resistivity hardly exhibits any change when the commensurate structural modulation sets in. Thus, the Fermi surface (FS)-driven mechanism behind the CDW formation [18] may not be at work here. More recently, a different $4 a \times 6 c$ CDW has been shown to occur in the surface of the $\mathrm{TaTe}_{4}$ crystals [19].

The origin of the CDW in $\mathrm{NbTe}_{4}$ and $\mathrm{TaTe}_{4}$ tetratellurides has been discussed for a long time. However, to the best of our knowledge there have not been first-principles studies discussing in detail the origin of their structural modulations until 


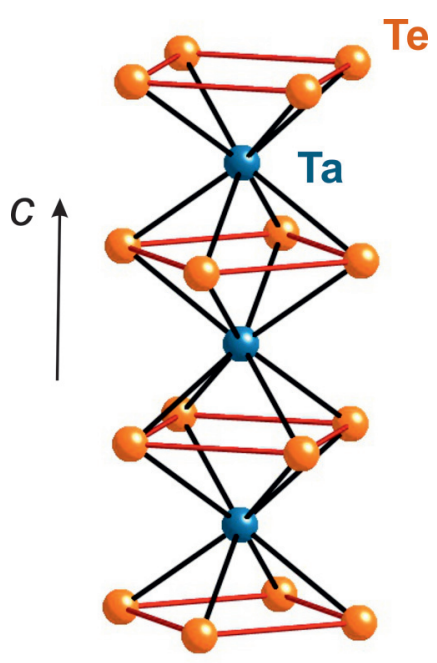

FIG. 1. Lateral view of a $\mathrm{TaTe}_{4}$ chain where the Ta atoms are in a square antiprismatic coordination.

very recently. Both di- and trichalcogenides have been the object of comparatively more attention. Previous theoretical work on these tetratellurides includes an early density functional theory (DFT) study by Bullet [20] noting the unusual strength of interchain interactions and a tight-binding study [16] proposing that FS nesting was indeed at work, although the reported FS does not provide compelling evidence for such claim. A more recent DFT study provided data concerning the electronic structure of $\mathrm{TaTe}_{4}$ but the origin of the bulk CDW was not considered [19]. Angular resolved photoemission spectra of $\mathrm{TaTe}_{4}$ exhibits a combination of 1D and 3D Fermi surface components [21]: The experiment does not clarify, though, if there are two decoupled Fermi surface components nor if the Fermi surface nesting plays a role in the structural modulation, although a recent theoretical study suggests that electron-phonon is the main source of the modulation [22]. As noted above, the electronic structure of the room temperature modulated structure has not been considered in any of these works.

Because of the renewed interest in these solids [4-7,19], we decided to carry out a first-principles DFT study for the average and modulated structures of $\mathrm{TaTe}_{4}$ by means of an analysis of the FS, electron-hole response function, and phonon spectra of the nonmodulated structure and structural optimizations of the CDW phase. We focus on $\mathrm{TaTe}_{4}$ although very similar arguments should apply for $\mathrm{NbTe}_{4}$. Recently, a study was reported concerning the Kohn anomaly in $\mathrm{TaTe}_{4}$ [22]. In the present paper we emphasize the analysis of the correlation between the structural and electronic features, which are the driving forces for the structural modulation. In particular, we show that the tellurium sublattice has considerable control of the structural instability even if the increase of metal-metal bonding could be thought to dominate the structural preference in these apparently strongly 1D materials. By doing this, we make clear that traditional electron-counting arguments can fail in dealing with the electronic instabilities of transition metal tellurides. In addition, we provide additional information concerning the nature of the phonon spectra, we consider in detail the distorted modulated structure, which was not considered in previous works, and we study the stability of alternative modulations.

\section{COMPUTATIONAL DETAILS}

The DFT calculations [23,24] were carried out using a numerical atomic orbitals DFT approach implemented in the SIESTA code [25-27]. The Perdew-Burke-Ernzerhof functional was used to account for the exchange-correlation energy [28]. The core electrons have been replaced by normconserving scalar relativistic pseudopotentials [29] factorized in the Kleinman-Bylander form [30]. We have used a splitvalence double- $\zeta$ basis set including polarization functions [31]. In all calculations, we used a cutoff of 800 Ry for the real space integrals and a tolerance of $10^{-4}$ and $10^{-3} \mathrm{eV}$ on the density matrix and the total energy, respectively, for the convergence of the SCF cycle. To sample the Brillouin zone (BZ) for the electronic states, a Monkhorst-Pack [32] $k$-point grid of $32 \times 32 \times 30$ was used for the nondistorted unit cell and scaled accordingly to the supercell calculations. The Lindhard electron-hole response function,

$$
\chi(q)=-\sum_{i, j} \sum_{k} \frac{f_{F}\left(\epsilon_{i}(k)\right)-f_{F}\left(\epsilon_{j}(k+q)\right)}{\epsilon_{i}(k)-\epsilon_{j}(k+q)},
$$

where $f_{F}$ is the Fermi-Dirac distribution function, was obtained from the computed DFT band eigenvalues $\epsilon_{i}(k)$. The integral over $k$ points of the $\mathrm{BZ}$ was approximated by a direct summation over a dense, regular grid of points. As the Lindhard function is more sensitive to the accuracy of the $\mathrm{BZ}$ integration than the total energy, the $k$-point grid used for its calculation must be more dense than in the standard self-consistent determination of the charge density and Kohn-Sham energy. The calculations were done using the eigenvalues obtained in the DFT calculation for the coarser grid, and interpolating their values in the denser grid, using a postprocessing utility available within the SIESTA package. In this paper, the BZ was sampled using a grid of $(200 \times 200 \times$ 200) $k$ points for the calculation of the Lindhard response function. Phonon calculations were carried out using the finite difference method available in the SIESTA code. In the case of the phonon band structure, a $k$-point grid of $10 \times 6 \times 10$ per minimum unit cell in a $5 \times 3 \times 5$ supercell was used. The Fermi-Dirac smearing was set to $5 \times 10^{-3} \mathrm{eV}$.

\section{CRYSTAL STRUCTURE AND ELECTRON COUNTING}

\section{A. Crystal structure}

$\mathrm{TaTe}_{4}$ crystallizes in a tetragonal structure with Ta atoms coordinated by eight $\mathrm{Te}$ atoms in a chainlike fashion. The structure of the $2 a \times 2 a \times 3 c$ commensurately modulated phase was solved by Bronsema et al. [12]. In the average structure determined by these authors, the Ta atoms are located in the center of a square antiprism of Te atoms, where the two $\mathrm{Te}_{4}$ square units are rotated $45^{\circ}$ with respect to each other as shown in Fig. 1. The crystal structure of $\mathrm{TaTe}_{4}$ is a tetragonal array of chains of this type (Fig. 2). The spacegroup symmetry of the average structure has been the matter of some discussion $[3,15,33]$ but it was finally determined by 


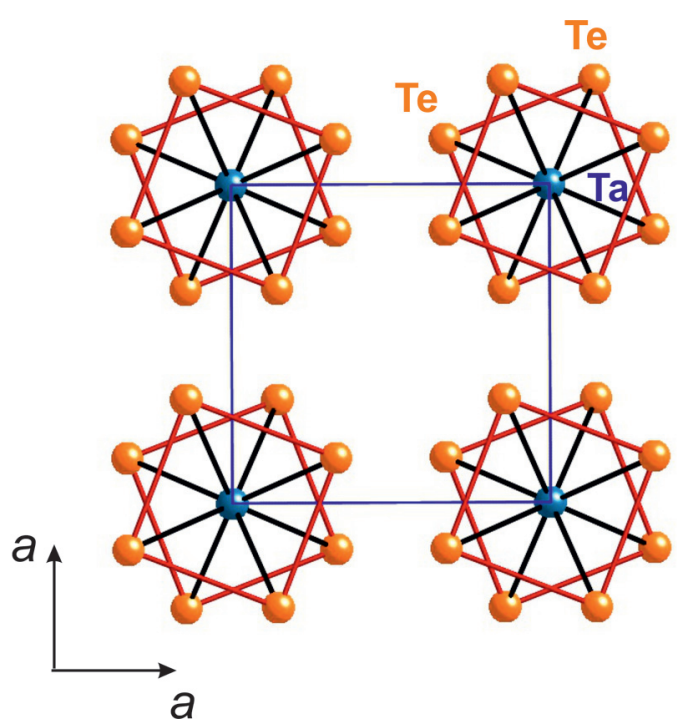

FIG. 2. Top view of the tetragonal structure of $\mathrm{TaTe}_{4}$. Te atoms are represented in orange and $\mathrm{Ta}$ in blue. Red lines represent Te-Te contacts in the shared faces of the square antiprisms.

Bronsema et al. [12] to be $P 4 / m c c$ with the lattice parameters $a=6.5154 \AA$ and $c=6.8118 \AA$. Their corresponding Wyckoff positions are $2 a$ for Ta atoms situated in $(0,0,1 / 4)$ and $(0,0,3 / 4)$ in fractional units, respectively $8 \mathrm{~m}$ for Te atoms situated in $(x, y, 0)$ and its equivalent positions, where $x=0.1438$ and $y=0.3274$. We carried out a relaxation of the internal coordinates while forcing the space-group symmetry, giving us $x=0.1501$ and $y=0.3296$, values which are in good agreement with the experimental ones. The space group of the $2 a \times 2 a \times 3 c$ commensurately modulated phase was found to be $P 4 / n c c$ [12]. Our DFT structural optimization is in excellent agreement with this work (see Sec. V). Let us note that weak superlattice reflections at $(1 / 2,0,1 / 3),(0$, $1 / 2,1 / 3)$, and very weak ones at $(0,0,1 / 3)$ were also observed $[12,15]$, although the last ones are most likely second harmonics satellites.

\section{B. Electron counting}

For a better understanding of the structural modulation, we need to determine how many electrons fill the Ta-based bands in the high temperature structure. The first thing to do is to look at the $\mathrm{Te}-\mathrm{Te}$ distances. Those within a $\mathrm{Te}_{4}$ square unit $(3.29 \AA)$ are shorter than the sum of the van der Waals radii of Te $(4.0 \AA)$, but too long to be considered as a real Te-Te bond (2.7-2.9 $\AA$ ). At careful inspection of the structure, we can observe that the shorter Te-Te contacts are not those in the $\mathrm{Te}_{4}$ square units but those connecting the squares of neighboring chains (the red bonds in Fig. 3, $2.93 \AA$ ), which are of the same order as many Te-Te single bonds. Under these considerations, the structure should be viewed as a 3D lattice of Ta-Te and Te-Te bonds as shown in Fig. 3. This is in contrast with the more usual description of this structure as a tetragonal array of $\mathrm{TaTe}_{4}$ chains (see Fig. 2) $[1,8,15]$. It is also in contrast with the $\mathrm{VS}_{4}$ structure $[10,11]$ where there are no short S-S contacts between the chains but within the chains (i.e., the short sides of the rectangular $\mathrm{S}_{4}$ groups separating the

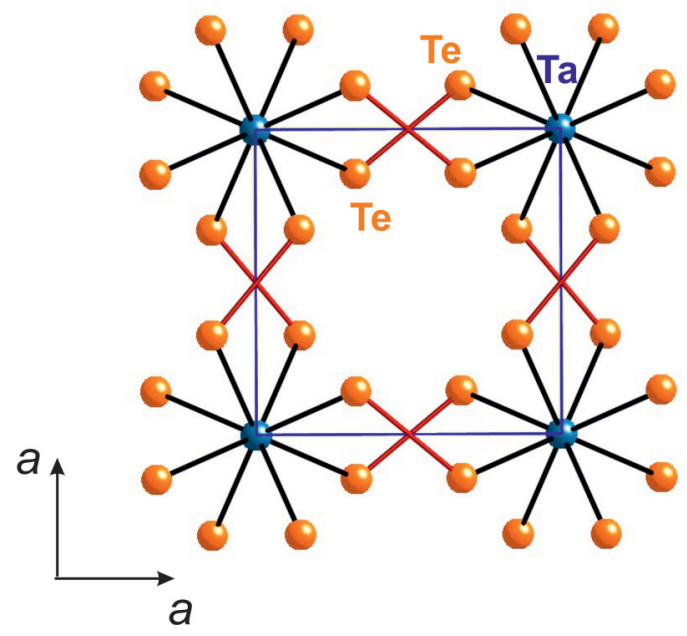

FIG. 3. Top view of the tetragonal structure of $\mathrm{TaTe}_{4}$. Te atoms are represented in orange and $\mathrm{Ta}$ in blue. Red lines represent the shorter Te-Te bonds of the structure $(2.93 \AA$ in the average crystal structure [12]).

successive V atoms, see Fig. S2 in the Supplemental Material (SM) [34]).

Because of the occurrence of Te-Te bonds, the tellurium atoms should be considered as $\left(\mathrm{Te}_{2}\right)^{2-}$, which means that only one electron is left to fill the Ta-based bands (i.e., Ta atoms are formally $d^{1}$ ). Thus, only the bottom Ta $5 d$-based bands may be partially filled. The $d$ orbitals of a transition metal in this coordination environment are such that the lowest energy orbital is a $d_{z^{2}}$ orbital and slightly higher in energy are the two $d_{x y}$ and $d_{x^{2}-y^{2}}$ orbitals. These two orbitals will be very weakly dispersive along the Ta chain direction whereas the $d_{z^{2}}$ orbital creates strong interactions and will thus lead to a wide band. Consequently, the bottom Ta-based band will be a dispersive $5 d_{z^{2}}$ band. Since there is just one electron to fill this band, the bottom part of the Ta-based bands will most likely be a half-filled Ta $5 d_{z^{2}}$ band.

On the basis of this formal electron count, we can predict that if the solid behaves as a 1D system around the Fermi level, it should be unstable to some kind of dimerization that would open a gap at the Fermi level, as is the case for $\mathrm{VS}_{4}[10]$. However, this is in conflict with experimental observations. To begin, the modulated structure is metallic. Moreover, the observed superlattice spots all have a $1 / 3$ component along the $c$ direction (the direction of the Ta chains). Thus, if the system can be treated as electronically 1D, the Ta band should not be half filled but one-third or two-thirds filled.

The latter possibility can be easily understood: It simply means that there is some electron transfer from the Te valence band toward the Ta $5 d_{z^{2}}$ band. This is indeed likely because of the short $\mathrm{Te}-\mathrm{Te}$ contacts between the $\mathrm{Te}_{2}$ units which may raise the top of the Te valence band. Thus, the top of the valence band may end up being higher than the Fermi level, transferring electrons to the Ta $5 d_{z^{2}}$ band which can thus become two-thirds filled.

In contrast, the first possibility may at first appear to be unlikely because, according to the occurrence of $\left(\mathrm{Te}_{2}\right)^{2-}$ units, the Te valence band is already full and cannot accept electrons 


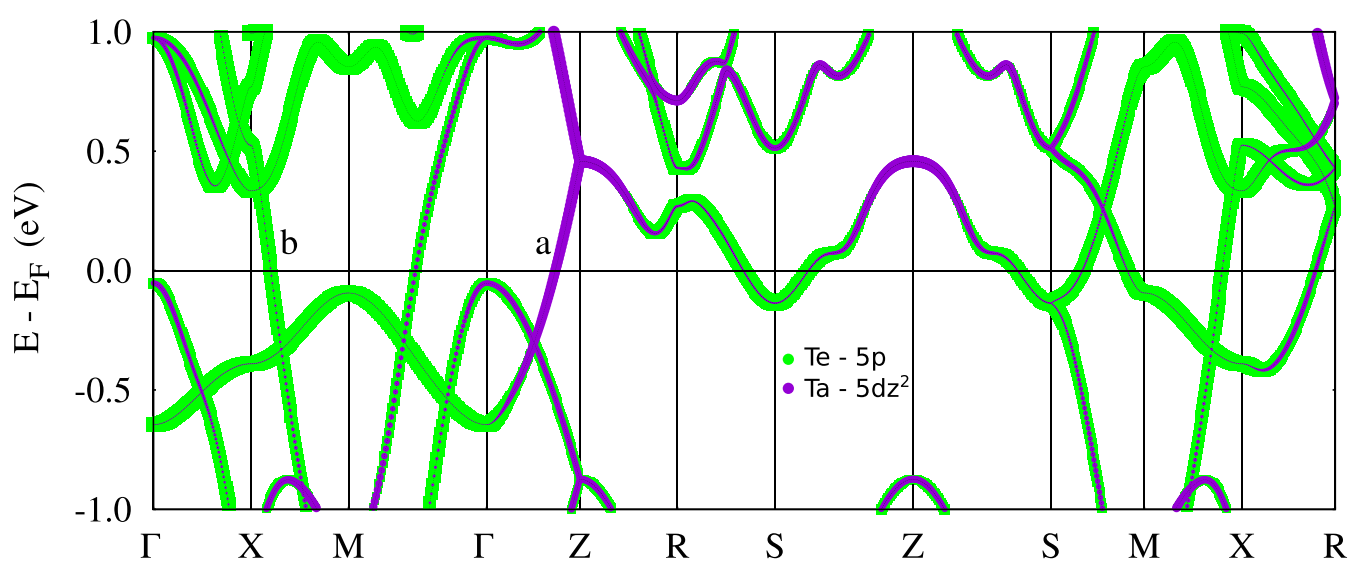

FIG. 4. Calculated band structure of the $\mathrm{TaTe}_{4}$ average structure [see Fig. 5(d) for the labeling of the special points]. The size of the green and purple dots are proportional to the Te $5 p$ and Ta $5 d_{z^{2}}$ character, respectively.

from Ta. To acquire electrons from the Ta atoms, part of the $\mathrm{Te}-\mathrm{Te}$ bonds should be broken, generating two $\mathrm{Te}^{2-}$, which is not the case according to the crystal structure [12]. However, let us remember that although the Te-Te contacts of the $\mathrm{Te}_{4}$ squares are longer than those between the squares, they are considerably shorter than the sum of the van der Waals radii. Taking into account both types of Te-Te contacts, one can see that one ( $a a$ ) plane of Te atoms is made of a series of strongly interacting Te-Te bonds (see Fig. S1 in the SM) so there must be very strong interactions between the Te-Te bonds along the layer. In that way, the empty band originating from the antibonding $\sigma^{*}$ Te-Te levels will broaden and the bottom part could overlap with the filled $5 d$ levels. The result would be a $\mathrm{Ta}$ to Te electron transfer, leading to a less than half-filled Ta $5 d_{z^{2}}$ band.

The previous considerations raise some serious questions impinging directly on the CDW mechanism: Do the TeTe short interchain contacts confer the electronic structure around the Fermi level with a 2D or 3D character? In that case, the probability to have good Fermi surface nesting is unlikely and the CDW would not be Fermi surface nesting driven. In addition, it is doubtful that the electronic transfer leads to a commensurate filling of the $5 d_{z^{2}}$ band. This objection can be somewhat dismissed by noting that the isostructural and isoelectronic $\mathrm{NbTe}_{4}$ undergoes a series of incommensurate modulations along the $c$ direction before becoming commensurate at low temperature. Thus, maybe in $\mathrm{TaTe}_{4}$ (but not in $\mathrm{NbTe}_{4}$ ) the electron transfer is very near the commensurability. Note also that whatever is the sense of the electron transfer, even if the Ta-based bands lead to a Fermi surface nesting driven CDW and thus to the opening of a gap at the Fermi level, the Te-based bands may not be necessarily affected, so the metallic character of the bands can be kept after the CDW sets in the material, as is in fact observed. Thus, it is not at all clear from simple electron counting arguments that the modulation exhibited by $\mathrm{TaTe}_{4}$ originates from a Fermi surface nesting phenomena, as in other metallic low-dimensional materials, or from a phonon driven instability, as, for instance, in $2 \mathrm{H}-\mathrm{NbSe}_{2}$ [35].

\section{ELECTRONIC STRUCTURE OF THE NONMODULATED STRUCTURE}

\section{A. Bands crossing the Fermi level}

The calculated band structure for the nonmodulated structure of $\mathrm{TaTe}_{4}$ is shown in Fig. 4. To understand the relationship with the crystal structure, we have decorated each band with green and purple dots of a size proportional to the Te $5 p$ and Ta $5 d_{z^{2}}$ character. This weighted band dispersion analysis reveals that there are essentially two types of bands crossing the Fermi level. The band crossing from $\Gamma$ to $Z$ (noted $a$ in the figure) originates from Ta $5 d_{z^{2}}$ orbitals and is actually a folded band because there are two Ta atoms in the unit cell related by a screw axis along the $c$ direction. Since the lower band component is only partially filled, it appears that the formal electron count for Ta is lower than $d^{1}$. Note also that the Te content of this pair of bands is quite sizable and in several lines of Fig. 4 even seems to dominate. However, this is partially due to the $1: 4$ stoichiometry which increases the total weight of the Te orbitals. What is, however, quite clear is that whereas these Ta $5 d_{z^{2}}$ bands are strongly dispersive along the $c$ direction, they are also engaged in interchain interactions (see, for instance, the $\Gamma-M-X-\Gamma$ lines in Fig. 4) because of the mixed $\mathrm{Ta}$ and $\mathrm{Te}$ character and the short interchain Te-Te contacts highlighted in Fig. 3. This band should thus have a strong memory of the 1D-type interactions associated with the Ta $5 d_{z^{2}}$-Ta $5 d_{z^{2}}$ interactions along the chain while exhibiting a non-negligible warping due to the tellurium content and the interchain interactions.

There is a second band crossing the Fermi level (noted $b$ in Fig. 4), which is mostly based on Te $5 p$ orbitals and exhibits a large dispersion on both directions, perpendicular (see $\Gamma-M$ and $M-X$ in Fig. 4) and parallel (see $M$-S in Fig. 4) to the $\mathrm{TaTe}_{4}$ chains. This mostly Te-based band should thus have a 3D character. In fact, the two bands overlap and interact quite strongly, thus interchanging character along different parts of the BZ (see, for instance, the Z-S line in Fig. 4). Thus, one should expect a relatively complex FS resulting from the interaction between the Ta $5 d_{z^{2}}$ and Te-based bands for nonmodulated $\mathrm{TaTe}_{4}$. 

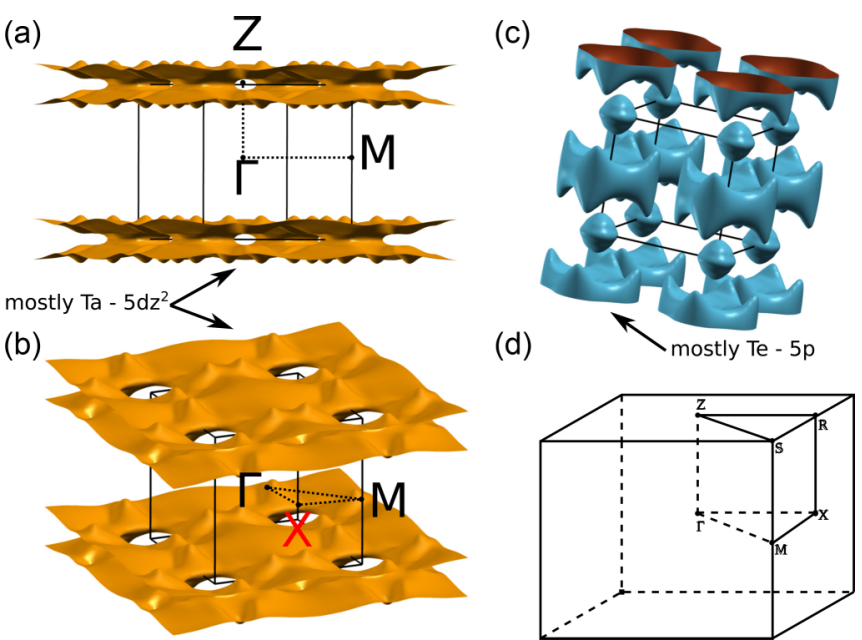

FIG. 5. Fermi surface of the $\mathrm{TaTe}_{4}$ average structure. (a) and (b) are two views of the component mostly originating in the Ta $5 d_{z}^{2}$ orbitals. (c) Component originating in the Te $5 p$ orbitals. (d) Brillouin zone (BZ) for a tetragonal lattice appropriate for $\mathrm{TaTe}_{4}$. The high symmetry points have the following coordinates: $\quad \Gamma=(0,0,0), X=(0.5,0,0), M=(0.5,0.5,0), Z=$ $(0,0,0.5), R=(0.5,0,0.5)$, and $S=(0.5,0.5,0.5)$ in units of the reciprocal lattice vectors.

\section{B. Fermi surface and electron-hole response}

The FS of $\mathrm{TaTe}_{4}$ is shown in Fig. 5. It is mostly made of two different contributions originating from the two types of bands $(a$ and $b)$ mentioned above. As expected, one of the components is relatively flat [Figs. 5(a) and 5(b)], although bearing a very substantial degree of warping and even holes. In fact, the two sheets making this portion of the FS touch at many points of the BZ, thus making a complex slab with holes and closed empty regions inside. Coming to the second component of the FS, we notice that it has a three-dimensional topology since the band generating this FS component crosses the Fermi level in several directions like $\Gamma-M$ and $M-X$ but also along $M$-S, where the band mixes with the Ta-based band. In fact, because of the degeneracy of the bands in the outer plane of the BZ (see the traject Z-R-S-Z in Fig. 4), there is a third Te $5 p$-based band crossing the Fermi level, leading to the closed almost spherical pockets around the corners of the BZ (point $S)$ in Fig. 5(c).

The warped FS component of Fig. 5(a), which contains substantial flat portions, could lead to non-negligible nesting and thus be at the origin of the CDW. This FS component, originating from band $a$ in Fig. 4, is associated with less than two electrons per unit cell (which is the filling associated with Ta atoms with an electron counting of $d^{1}$ ). This result confirms that, in contrast with the usual situation in many transition metal tellurides, there has been a formal electron transfer from the $\mathrm{Ta}$ to the Te sublattices, i.e., the formal electron counting for Ta is $d^{1-\delta}$ with $\delta \sim 0.2$, in between those of a one-third filled $(\delta=2 / 3)$ and half-filled $(\delta=0)$. It is because of this transfer that the double pillow pockets of Fig. 5(c) are created. As mentioned above, this reverse electron transfer means that the tellurium sublattice cannot be considered as a series of isolated (i.e., not directly interacting)
Te-Te bonds (i.e., $\left(\mathrm{Te}_{2}\right)^{2-}$ ). A series of Te-Te bonds is only compatible with $\delta=0$. The filling of additional Te-based levels implicates that some of the antibonding and thus empty Te-Te levels have been stabilized so as to occur in the region of the Fermi level and are thus filled. This can occur when the Te-Te interaction is considerably delocalized through the tellurium sublattice because then, the Te-Te empty antibonding levels interact and spread into wide bands because of the direct Te-Te interbond interactions, the bottom part of such bands (which have some bonding character between the Te-Te units, i.e., the dotted lines in Fig. S1 of the SM) overlaps with filled transition metal levels, leading to a reverse electron transfer from the transition metal to tellurium (and to some bonding between Te-Te bonds). A consequence of these electron transfers is that the formal electron counting for the tellurium atoms and fragments of transition metal tellurides is sometimes not quite obvious [36,37]. As a consequence of these transfers, the anion sublattice of transition metal tellurides often contains tellurium fragments larger than dimers and even large polianionic units $[37,38]$. The present results clearly confirm that the tellurium layers of $\mathrm{TaTe}_{4}$ should be regarded as a strongly interconnected lattice of $\mathrm{Te}_{2}$ units, as sketched in Fig. S1 (SM).

To clarify if the structural modulation originates in a Fermi surface nesting phenomena, we have performed Lindhard function calculations across several planes in both $\left(a^{*} a^{*}\right)$ and $\left(a^{*} c^{*}\right)$. According to the experimental findings, if the CDW modulation originates from FS nesting, we should find sharp maxima in the Lindhard function calculations for $1 / 2 a^{*}$ and $1 / 3 c^{*}$ components, i.e., in the corners of Fig. 6(b). However, this is not the case. For the $\left(a^{*} a^{*}\right)$ planes, when we vary the $q_{c^{*}}$ component, the maximum in the Lindhard function occurs for $q_{c^{*}}=1 / 2$ [Fig. 6(c)] and not 1/3. Even so, the occurring maximum is not a typical logarithmic dispersion for a Fermi surface nesting driven instability, but it covers a rather broad region of suitable nesting vectors for $q_{a^{*}}$ in the $[0.1-0.5]$ interval. It could be argued that the $1 / 2$ components of the modulation along the interchain directions originate from Coulomb coupling between intrachain modulations. However, in the case where we calculate the Lindhard function for $\left(a^{*} c^{*}\right)$ planes and vary the second $q_{a^{*}}$ component [Figs. 6(d)6(f)], we find that the maximum response is given by $q=$ $(0,0,0.39)$ [see Fig. 6(d)]. This wave vector does not correspond to the $2 \mathrm{k}_{F}$ value of a folded $d_{z^{2}}$ band for Ta $\sim d^{0.8}$, i.e., is not related to the Ta $5 d_{z^{2}}$-based FS component of Fig. 5(a). In fact, this wave vector is associated with an interband nesting and would lead to an incommensurate modulation along the $c$ direction, which is not a proper nesting vector to explain the observed modulation. We thus may dismiss the possibility of a FS-driven instability, leading to the structural modulation manifested in $\mathrm{TaTe}_{4}$.

\section{STRUCTURAL MODULATION}

Assuming no prior knowledge of the modulated structure of this material, the clear-cut answer of phase stability/ordering, whichever they might be, is provided by the energy gain of each phase with respect to the undistorted 

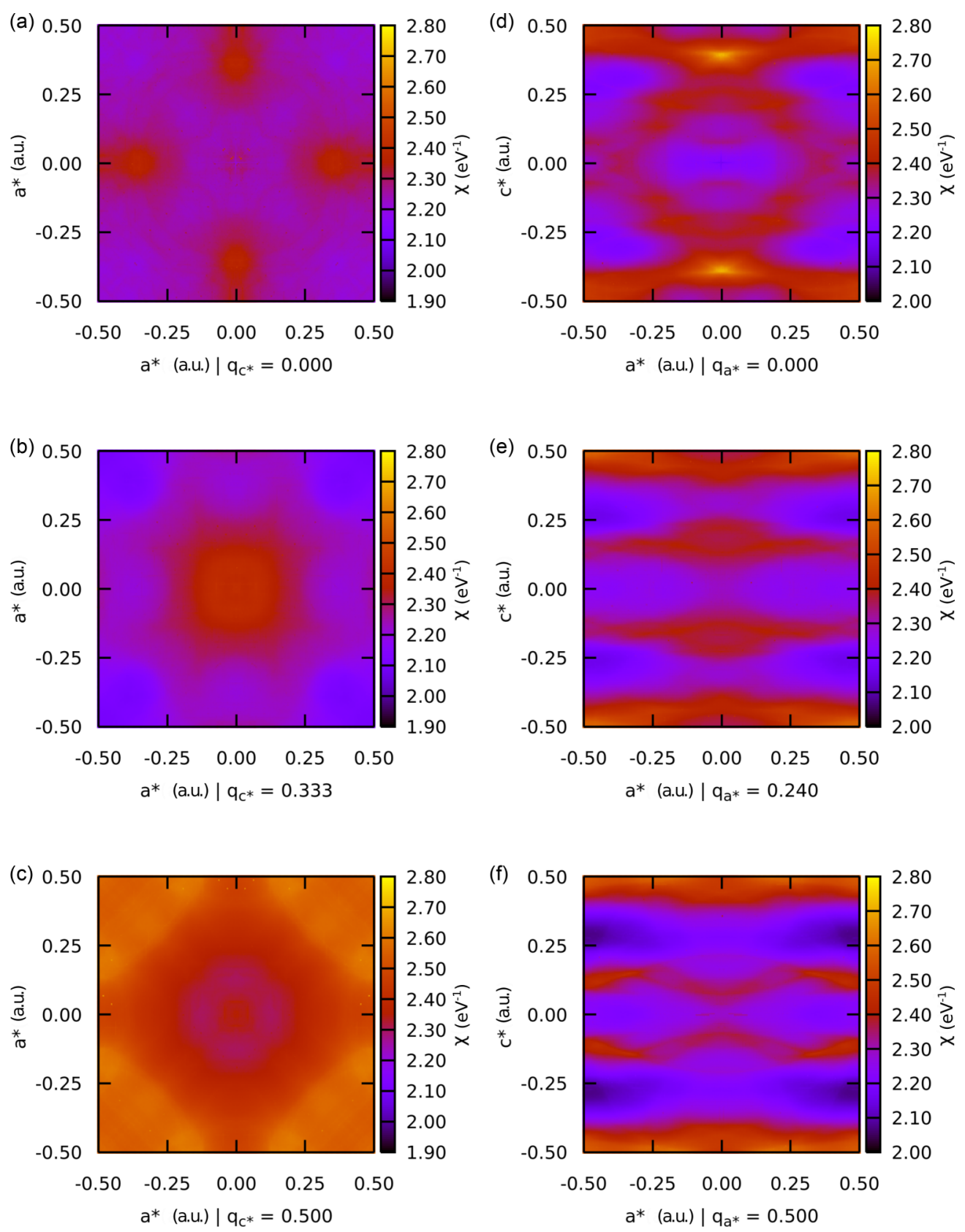

FIG. 6. 2D mapping of the calculated Lindhard function of $\mathrm{TaTe}_{4}$ in the $\mathrm{a}^{*}-\mathrm{a}^{*}$ plane for different $q_{c^{*}}$ components (a)-(c), and in the $a^{*}-c^{*}$ plane for different secondary $q_{a^{*}}$ components (d)-(f). The bars represent the Lindhard function value in arbitrary units.

structure. In this section, we examine this point by calculating the phonon dispersion and carrying out actual structural optimizations for different modulated structures.

\section{A. Phonon band structure}

The vibrational modes in the undistorted phase can give information to understand the origin of the possible structural modulations. The calculated phonon band structure is shown in Fig. 7. Several imaginary phonon modes can be identified, with minima not too far from $\left(0,0, \frac{1}{4}\right),\left(\frac{1}{2}, \frac{1}{2}, \frac{1}{3}\right)$, $\left(0, \frac{1}{2}, \frac{1}{3}\right)$. These soft-phonon modes (corresponding to negative eigenvalues of the harmonic dynamical matrix) are an indication of a structural instability, and a possible lowering of the system's energy can be obtained via atomic displacements along the corresponding vibrational mode. Indeed, this complex phonon band structure is a consequence of a landscape of lattice instabilities with a valley of modulations along the $c^{*}$ axis which is between $\frac{1}{4}$ and $\frac{1}{3}$ [see Fig. 7(b)], indicating that multiple possible phases might coexist (and compete) at low temperatures. Guided by the soft-phonon minima, we have performed internal coordinate relaxations for each of the possible phases, and the lowest energy state we obtained was the $2 a \times 2 a \times 3 c$ phase, in agreement with experimental observations. All the other stable phases were higher in energy than the $2 a \times 2 a \times 3 c$ phase as presented in Table I. An optimization for the $1 a \times 1 a \times 3 c$ phase led to the average 


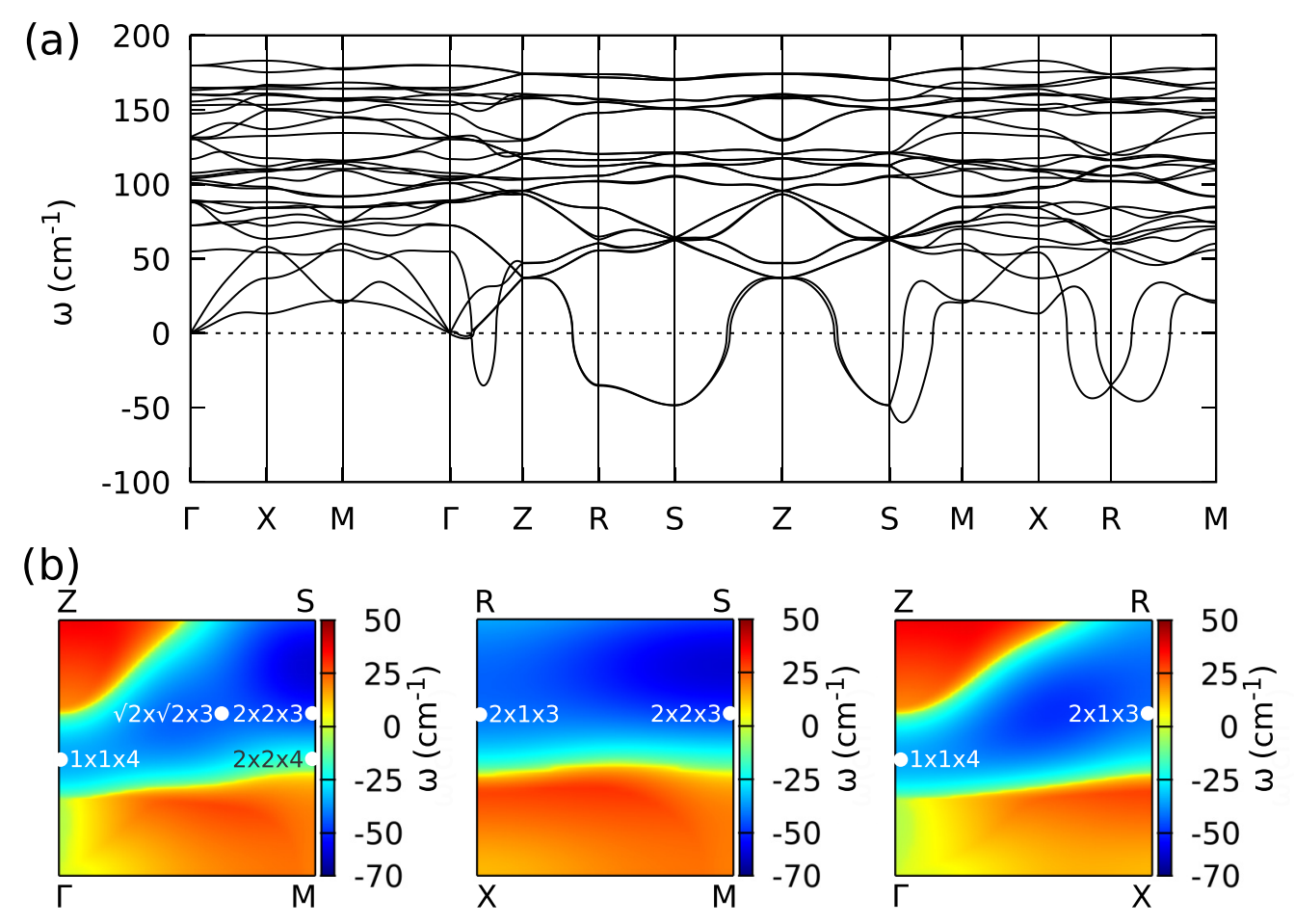

FIG. 7. Phonon band structure along high-symmetry lines (a). Landscape of soft-phonon frequencies along high-symmetry planes in reciprocal space. The lowest energy phonon mode for each $k$ point is represented (b). The wave vectors of the different commensurate modulated structures considered in Table I are shown as white dots in (b).

structure. We also considered a $\sqrt{2} a \times \sqrt{2} a \times 3 c$ phase. The energy gain for this structure was found to be half that of the $2 a \times 2 a \times 3 c$ phase; this is consistent with the fact that this modulation is only found for temperatures higher than $450 \mathrm{~K}$.

\section{B. Modulated structure}

The CDW structure and lattice symmetry have been determined experimentally by Bronsema et al. [12]. It was later restated by Corbett et al. [39] that the modulated structure symmetry is indeed P4/ncc. We present in Table S-I of the SM a comparison between the experimental structure and our calculated structure of the lowest energy state. The calculated structure is in excellent agreement with the experimental one.

The calculated $2 a \times 2 a \times 3 c$ phase as well as the $2 a \times$ $1 a \times 3 c$ one can be described on the basis of Fig. 8. The unit cell of both phases contains two different $\mathrm{TaTe}_{4}$ chains with different trimerization patterns labeled A and B. In chain A [Fig. 8(b)], three different Ta-Ta distances are generated (see Table II); two are shorter than in the average structure and

TABLE I. Energy gain of different commensurate superstructures with respect to the $\mathrm{TaTe}_{4}$ average structure.

\begin{tabular}{lc}
\hline \hline Periodicity & Energy gain (meV/f.u.) \\
\hline $1 a \times 1 a \times 4 c$ & -13 \\
$\sqrt{2} a \times \sqrt{2} a \times 3 c$ & -28 \\
$2 a \times 2 a \times 4 c$ & -31 \\
$2 a \times 1 a \times 3 c$ & -38 \\
$2 a \times 2 a \times 3 c$ & -57 \\
\hline \hline
\end{tabular}

one is longer, whereas in chain B [Fig. 8(c)], there are only two different distances; the two shorter Ta-Ta distances are identical in that case but different in chain A. The average value of the two shorter distances in chain $\mathrm{A}(3.128 \AA)$ is very similar to the short distance in chain $\mathrm{B}(3.133 \AA)$. Trimerized units or clusters are thus formed in every chain, increasing the metal-metal bonding. Interestingly, the atomic displacements corresponding to these two types of chains can be inferred from linear combinations of the soft-phonon modes obtained for $\mathrm{q}_{1}=\left(\frac{1}{2}, \frac{1}{2}, \frac{1}{3}\right)$ and $\mathrm{q}_{2}=\left(\frac{1}{2},-\frac{1}{2}, \frac{1}{3}\right)$. In particular, $\mathrm{q}_{1}+\mathrm{q}_{2}$ gives the distortion pattern for chain $\mathrm{B}$, while $\mathrm{q}_{1}-\mathrm{q}_{2}$ gives that of chain A. This mixing of modes also explains why there are two different types of chains coming from only one softphonon branch in the vibrational band structure. We can infer from these calculations that the CDW modulation of $\mathrm{TaTe}_{4}$ originates from a phonon instability.

Although as shown in Figs. 8(b) and 8(c) the structural variations are strongly located on the Ta sublattice, the occurrence of these triplets induces a response of the Te sublattice. There are two different types of Te squares (they are really squares in chain A but rectangles in chain B): one-third of them have shorter Te-Te contacts whereas two-thirds have longer Te...Te contacts (see Table III). Those being contracted are those in between Ta atoms which have moved apart; this is easy to understand since the $\mathrm{TaTe}_{4}$ chains tend to keep the Ta-Te bonding. The variation of these Te-Te distances is considerably larger (one order of magnitude) than the variation of the Te-Te interchain bonds (see Table III) which remain relatively constant. Thus, it is clear that the system tries to keep the Te-Te bonds as stable as possible and, in particular, to keep the strong Te-Te interchain bonds. The rotations and shifts undergone by the Te atoms under the modulation have 


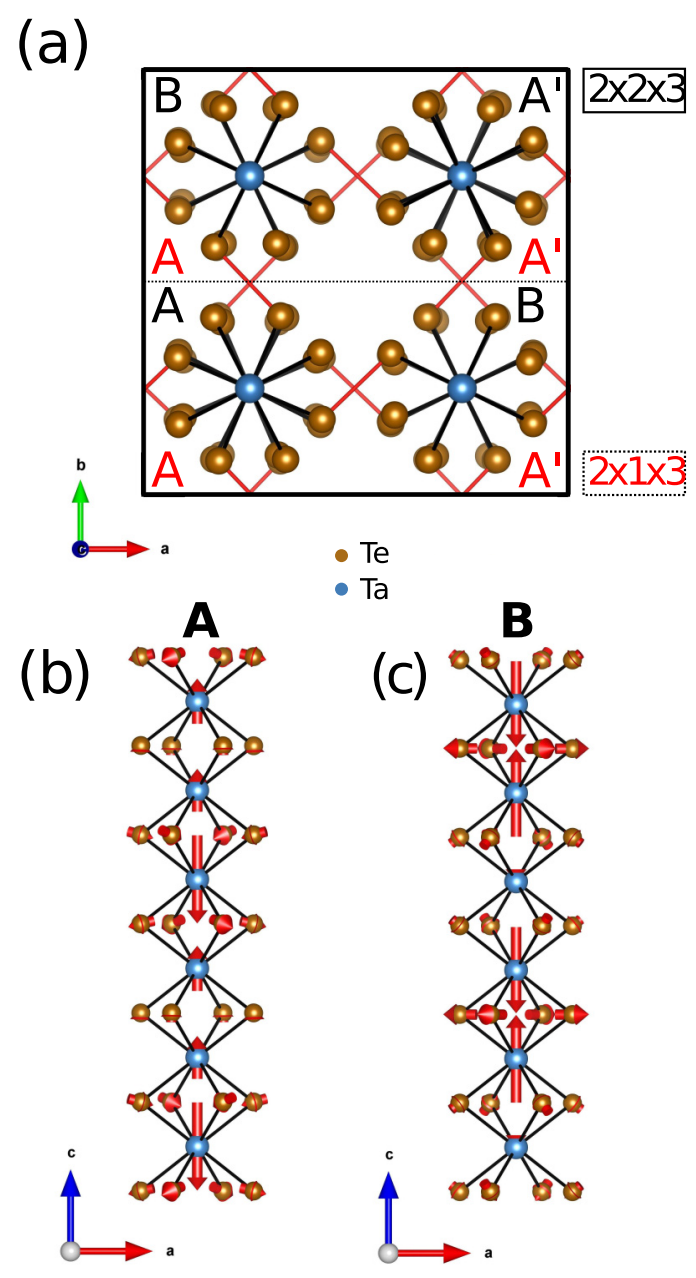

FIG. 8. (a) Modulated chain arrangement in the $2 \times 2 \times 3$, respectively, $2 \times 1 \times 3$ supercells. The black $/$ red chain labels are those associated with the $2 \times 2 \times 3 / 2 \times 1 \times 3$ supercells. (b), (c) Unstable phonon modes correspondence to each chain type in the $2 \times 2 \times 3$ and $2 \times 1 \times 3 \mathrm{TaTe}_{4}$ supercells.

this purpose. The variation of the Ta-Te bonds (Table IV) can be analyzed in the same way. All structural variations noted for the optimized structures mirror those of the experimental ones. In summary, it appears that optimization of the metalmetal bonding subject to inducing the minimum alteration of the Te sublattice is the driving force for the CDW irrespective of the nature of the Fermi surface.

A Mulliken population analysis mirrors such discussion: A slight increase of the overlap population associated with

TABLE II. Ta-Ta contacts in the optimized average structure and in the two types of chains in the optimized modulated structure. Note that the distortion is allowed within the experimental lattice parameters. Refer to Fig. 9 for labels.

\begin{tabular}{lcccc}
\hline \hline$d(\AA)$ & Average & Type A & Type B & No. \\
\hline Ta-Ta & \multirow{2}{*}{3.406} & 3.156 & 3.952 & (a) \\
& & 3.101 & 3.133 & (b) \\
& & 3.960 & 3.133 & (c) \\
\hline \hline
\end{tabular}

TABLE III. Te-Te contacts in the optimized average structure and in the two types of chains in the optimized modulated structure. Both the interchain and the intrachain distances are included. A particular case represents chain-type B where the square symmetry is broken and we have two types of distances within the rectangle. Moreover, the planes containing these two consecutive rectangles are rotated by $90^{\circ}$ with respect to each other (thus the order of writing in the table). Note that the distortion is allowed within the experimental lattice parameters. Refer to Fig. 9 for labels.

\begin{tabular}{ccccccc}
\hline \hline$d(\AA)$ & Average & Interchain & Average & Square A & Square B & No. \\
\hline Te-Te & 2.959 & 2.974 & 3.337 & 3.143 & $3.389-3.471$ & $\mathrm{~S}_{a}$ \\
& & 2.965 & & 3.414 & $3.471-3.389$ & $\mathrm{~S}_{b}$ \\
& 2.929 & & 3.447 & 3.146 & $\mathrm{~S}_{c}$ \\
\hline \hline
\end{tabular}

the Ta-Ta bonds is only partially balanced by a smaller decrease of the Te-Te overlap populations within the Te squares. The Ta-Te and Te-Te intersquare overlap populations do not clearly distinguish between the two structures. However, the differences are small and given the well-known possible shortcomings of the Mulliken analysis when dealing with atoms with very diffuse orbitals, we prefer to focus on more topologically robust indices like the Hirshfeld or Voronoi atomic populations. The average Hirshfeld atomic charges (in $e$ units) for $\mathrm{Ta} / \mathrm{Te}$ in the average and modulated structures are $-0.054 /+0.013$ and $-0.063 /+0.016$, respectively. The differences are indeed small but they clearly prove an increase in the Ta electron density upon the modulation. Such a shift of electron density from the Te to the Ta atoms is indeed what is expected for a system where there is an increase in Ta-Ta bonding at the expense of Te-Te bonding. When going deeper into the analysis, it becomes clear that the Ta atoms associated with the shorter Ta-Ta bonds are those associated with higher negative charge: -0.066 and -0.065 versus -0.059 in the A chains as well as -0.065 and -0.065 versus -0.061 in the $\mathrm{B}$ chains. Note that the shortest Ta-Ta bond (3.101 $\AA$ ) occurs in chain A and is associated with the Ta with the larger negative charge (-0.066). Exactly the same analysis holds for the Voronoi charges. It is interesting to note that the next stable superstructure, the $2 a \times 1 a \times 3 c$ one, which contains just two chains in the new unit cell and thus has less freedom to allow the Ta atoms to move, contains trimerized chains where the shorter Ta-Ta bonds are a bit longer than in the $2 a \times 2 a \times 3 c$ superstructure $(3.145 / 3.156$ versus $3.101 / 3.156 \AA)$ and the

TABLE IV. Ta-Te contacts in the optimized average structure and in the two types of chains in the optimized modulated structure. Note that the distortion is allowed within the experimental lattice parameters. Refer to Fig. 9 for labels.

\begin{tabular}{ccccc}
\hline \hline$d(\AA)$ & Average & Type A & Type B & No. \\
\hline Ta-Te & \multirow{2}{*}{2.910} & 2.845 & 2.969 & 1 \\
& & 2.982 & 2.843 & 2 \\
& 2.971 & 2.945 & 3 \\
& 2.836 & 2.926 & 4 \\
& 2.935 & 2.839 & 5 \\
& 2.934 & 2.982 & 6 \\
\hline
\end{tabular}




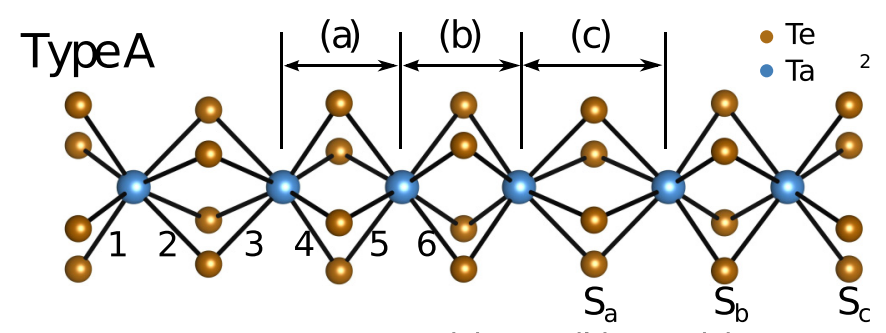

Type B

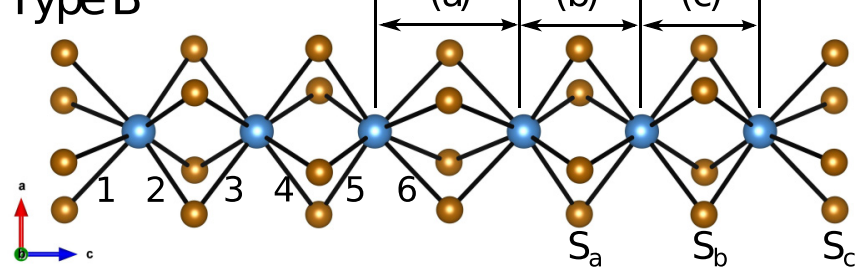

FIG. 9. Distance labels in the different modulated chains. Labels 1-6 refer to Ta-Te distances as described in Table IV, Ta-Ta distances labeled as (a), (b) and (c) described in Table II, respectively, labels $\mathrm{S}_{a}-\mathrm{S}_{c}$ corresponding to Te-Te contacts as described in Table III.

longer Ta-Ta bonds are a bit shorter (3.913 versus $3.960 \AA$ ), i.e., the trimerization is a bit weaker. The larger strain in the $2 a \times 1 a \times 3 c$ superstructure is reflected in a slight increase of the interchain Te-Te bonds: 2.970 versus $2.956 \AA$ in the $2 a \times 2 a \times 3 c$ superstructure. In summary, the combined analysis of the structural and electronic changes in the different superstructures confirms that the driving force for the ground-state modulation is the optimization of the Ta$\mathrm{Ta}$ bonding while inducing the minimum decrease in $\mathrm{Te}-\mathrm{Te}$ bonding; although such structural rearrangement is associated with relatively large individual bond-length changes, there is a subtle compensation of energy changes such that the structural transition occurs with a very small energy variation.

\section{Electronic structure}

Using this structure, we have calculated the band structure of the CDW phase. As was also found experimentally, the modulated phase is clearly metallic, as one can see in Fig. 10. The extensive folding leads to the four components of the Fermi surface shown in Fig. 11. It is clear from this
Fermi surface that $\mathrm{TaTe}_{4}$ in the CDW structure is a 3D metal, in agreement with the experimental observation of $\rho_{c} / \rho_{a} \sim 1[4-7,17]$. Since $\mathrm{TaTe}_{4}$ is already in the modulated phase at room temperature, it is not possible to know the resistivity change when the transition occurs. However, for $\mathrm{NbTe}_{4}$, which is structurally and electronically very similar, the change in the resistivity is very small without variation of the anisotropy [17]. This is also consistent with the 3D character of the FS calculated for the average and modulated structures of $\mathrm{TaTe}_{4}$. However, the general shape of the FS has been strongly affected by the modulation. The different components of the Fermi surface in Fig. 11 contain numerous closed circuits which are consistent with recent studies reporting a very large magnetoresistance for $\mathrm{TaTe}_{4}$ in the CDW state [4-7]. We note the occurrence of a crossing between a steeply rising band with almost linear dispersion and another less dispersive band at the Fermi level near the $M$ point (see the $M$ to $X$ direction in Fig. 11). Inclusion of spin-orbit coupling does not alter this situation. The two bands have mixed Ta and $\mathrm{Te}$ character and the crossing is a consequence of the structural modulation. This feature is reminiscent of the observation of very large linear magnetoresistance for materials exhibiting a Dirac cone at the Fermi level $[40,41]$ and could be at the origin of very large magnetoresistance exhibited by $\mathrm{TaTe}_{4}$.

Figure 10 is in strong contrast with the usual shape of the band structure for metallic quasi-1D transition metal chalcogenides undergoing a structural modulation along the chain direction. As mentioned before, the crystal structure of $\mathrm{VS}_{4}$, although strongly related with that of $\mathrm{TaTe}_{4}$, contains $\mathrm{VS}_{4}$ chains which are the result of a dimerization within the chains (see Fig. S2 in the SM). This may be conceptually described as being the result of a usual Peierls dimerization of a chain of transition metal atoms $(M)$ possessing a half-filled quasi-1D $d_{z^{2}}$ band. Note that in the present case, since the repeat unit of the average cell already contains two Ta atoms because of the antiprismatic nature of the coordination, the distortion is formally a tetramerization. The band structure for such a system should exhibit a semiconducting gap separating two sets of transition-metal-based bands associated with bonding and antibonding $M-M$ levels resulting from the modulation. This is exactly what is observed in the case of the dimerized structure of $\mathrm{VS}_{4}$ (see Fig. S3 in the SM). Both $\mathrm{TaTe}_{4}$ and

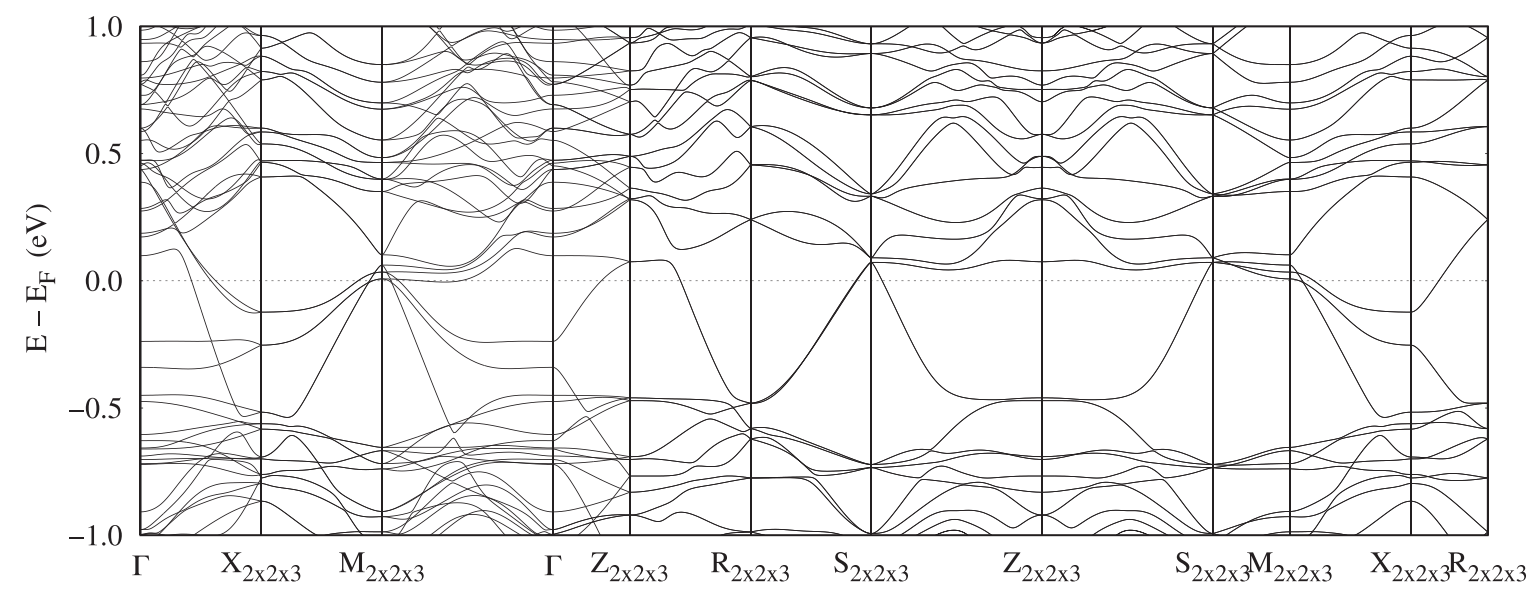

FIG. 10. Band structure for the $2 a \times 2 a \times 3 c$ modulated $\mathrm{TaTe}_{4}$ structure. 


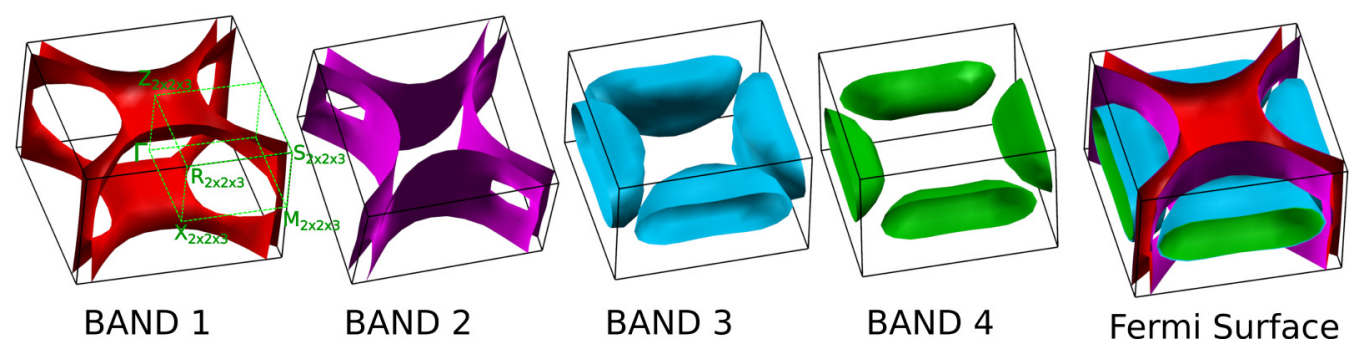

FIG. 11. Calculated Fermi surface (right) for the $2 a \times 2 a \times 3 c$ modulated $\mathrm{TaTe}_{4}$ structure with the contribution of the four different subbands shown with different colors.

$\mathrm{VS}_{4}$ contain two $X_{2}^{2-}(X=\mathrm{S}$ or $\mathrm{Te})$ chalcogen-chalcogen bonds (although of different nature), so the formal electron counting for the transition metal atom is the same in both compounds. However, the chalcogen-chalcogen bonds occur within the chains in $\mathrm{VS}_{4}$ but between the chains in $\mathrm{TaTe}_{4}$. This subtle structural difference completely changes the nature of the electronic structure and leads to a completely different low-temperature structural modulation and physical behavior.

\section{CONCLUSIONS}

We have discussed the electronic structure of $\mathrm{TaTe}_{4}$ and argued that, despite the occurrence of $\mathrm{TaTe}_{4}$ square antiprismatic chains in the crystal structure, this solid has a 3D electronic structure. This is primarily the result of the interchain coupling between adjacent chains through Te atoms. The Te-Te interchain distances in this compound are compatible with a Te-Te bond. Based on our FS and electron-hole response function calculations, we have dismissed a Fermi surface driven instability as the origin of the modulated structure. However, this mechanism could be invoked to explain the modulation of the related $\mathrm{VS}_{4}$ solid which does not exhibit strong interchain contacts. We have calculated the phonon dispersion and described the ordering of the possible modulated phases. The optimized $2 a \times 2 a \times 3 c$ structure is found to be the more stable, in agreement with experimental observations, and can be obtained directly from a soft-phonon mode computed for the undistorted structure. The nature of the distortions with respect to the average structure suggest that the driving force for the distortion is the maximization of Ta-Ta metal-metal bonding subject to inducing the minimum decrease in bonding within the Te sublattice.

\section{ACKNOWLEDGMENTS}

This paper was supported by Spanish MINECO (the Severo Ochoa Centers of Excellence Program under Grants No. SEV-2017-0706 and No. FUNFUTURE CEX2019000917-S), Spanish MICIU, AEI, and EU FEDER (Grants No. PGC2018-096955-B-C43 and No. PGC2018-096955-BC44), Generalitat de Catalunya (Grant No. 2017SGR1506 and the CERCA Programme), and the European Union MaX Center of Excellence (EU-H2020 Grant No. 824143). Computational resources have been provided partially by the supercomputing facilities of the Université Catholique de Louvain (CISM/UCL) and the Consortium des Équipements de Calcul Intensif en Fédération Wallonie Bruxelles (CÉCI) funded by the Fond de la Recherche Scientifique de Belgique (F.R.S.-FNRS) under Convention No. 2.5020.11 and by the Walloon Region.
[1] K. Selte and A. Kjekshus, Acta Chem. Scand. 18, 690 (1964).

[2] E. Bjerkelund and A. Kjekshus, Z. Anorg. Allgem. Chem. 328, 235 (1964).

[3] E. Bjerkelund and A. Kjekshus, J. Less Common Met. 7, 231 (1964).

[4] Y. Gao, L. Xu, Y. Qiu, Z. Tian, S. Yuan, and J. Wang, J. Appl. Phys. 122, 135101 (2017).

[5] X. Luo, F. C. Chen, Q. L. Pei, J. J. Gao, J. Yan, W. J. Lu, P. Tong, Y. Y. Han, W. H. Song, and Y. P. Sun, Appl. Phys. Lett. 110, 092401 (2017).

[6] X. Yang, Y. Zhou, M. Wang, H. Bai, X. Chen, C. An, Y. Zhou, Q. Chen, Y. Li, Z. Wang, J. Chen, C. Cao, Y. Li, Y. Zhou, Z. Yang, and Z.-A. Xu, Sci. Rep. 8, 6298 (2018).

[7] B. S. de Lima, N. Chaia, T. W. Grant, L. R. de Faria, J. C. Canova, F. S. de Oliveira, F. Abud, and A. J. S. Machado, Mater. Chem. Phys. 226, 95 (2019).

[8] A. Meerschaut and J. Rouxel, in Crystal Chemistry and Properties of Materials with Quasi-One-Dimensional Structures, edited by J. Rouxel (D. Reidel Publishing Company, Dordrecht, Holland, 1986), pp. 205-279.

[9] E. Canadell, S. Jobic, R. Brec, J. Rouxel, and M.-H. Whangbo, J. Solid State Chem. 99, 189 (1992).

[10] M. N. Kozlova, Y. V. Mironov, E. D. Grayfer, A. I. Smolentsev, V. I. Zaikovskii, N. A. Nebogatikova, T. Y. Podlipskaya, and V. E. Fedorov, Chem. Eur. J. 21, 4639 (2015).

[11] I. B. R. Allmann, A. Kutoglu, H. Roesch, and E. Hellner, Naturwissenschaften 51, 263 (1964).

[12] K. D. Bronsema, S. van Smaalen, J. D. Boer, G. A. Wiegers, F. Jellinek, and J. Mahy, Acta Crystallogr. B 43, 305 (1987).

[13] J. Kusz and H. Böhm, Acta Crystallogr. B 50, 649 (1994).

[14] S. van Smaalen, K. D. Bronsema, and J. Mahy, Acta Crystallogr. B 42, 43 (1986).

[15] F. W. Boswell, A. Prodan, and J. K. Brandon, J. Phys. C: Solid State Phys. 16, 1067 (1983)

[16] M.-H. Whangbo and P. Gressier, Inorg. Chem. 23, 1228 (1984).

[17] S. Tadaki, N. Hino, T. Sambongi, K. Nomura, and F. Lévy, Synth. Met. 38, 227 (1990). 
[18] G. Grüner, Density Waves in Solids, Frontiers in Physics, Vol. 89 (Addison-Wesley, Reading, MA, 1994).

[19] H. Sun, Z. Shao, T. Luo, Q. Gu, Z. Zhang, S. Li, L. Liu, H. Gedeon, X. Zhang, Q. Bian, J. Feng, J. Wang, and M. Pan, New J. Phys. 22, 083025 (2020).

[20] D. W. Bullet, J. Phys. C: Solid State Phys. 17, 253 (1984).

[21] F. Zwick, H. Berger, M. Grioni, G. Margaritondo, L. Forró, J. LaVeigne, D. B. Tanner, and M. Onellion, Phys. Rev. B 59, 7762 (1999).

[22] F.-H. Liu, W. Fu, Y.-H. Deng, Z.-B. Yuan, and L.-N. Wu, Appl. Phys. Lett. 119, 091901 (2021).

[23] P. Hohenberg and W. Kohn, Phys. Rev. 136, B864 (1964).

[24] W. Kohn and L. J. Sham, Phys. Rev. 140, A1133 (1965).

[25] J. M. Soler, E. Artacho, J. D. Gale, A. García, J. Junquera, P. Ordejón, and D. Sánchez-Portal, J. Phys.: Condens. Matter 14, 2745 (2002).

[26] E. Artacho, E. Anglada, O. Diéguez, J. D. Gale, A. García, J. Junquera, R. M. Martin, P. Ordejón, J. M. Pruneda, D. SánchezPortal, and J. M. Soler, J. Phys.: Condens. Matter 20, 064208 (2008).

[27] A. García, N. Papior, A. Akhtar, E. Artacho, V. Blum, E. Bosoni, P. Brandimarte, M. Brandbyge, J. I. Cerdá, F. Corsetti, R. Cuadrado, V. Dikan, J. Ferrer, J. Gale, P. García-Fernández, V. M. García-Suárez, S. García, G. Hihs, S. Illera, R. Korytár et al., J. Chem. Phys. 152, 204108 (2020).

[28] J. P. Perdew, K. Burke, and M. Ernzerhof, Phys. Rev. Lett. 77, 3865 (1996).
[29] N. Troullier and J. L. Martins, Phys. Rev. B 43, 1993 (1991).

[30] L. Kleinman and D. M. Bylander, Phys. Rev. Lett. 48, 1425 (1982).

[31] E. Artacho, D. Sánchez-Portal, P. Ordejón, A. García, and J. M. Soler, Phys. Status Solidi (b) 215, 809 (1999).

[32] H. J. Monkhorst and J. D. Pack, Phys. Rev. B 13, 5188 (1976).

[33] D. J. Eaglesham, D. Bird, R. L. Witners, and J. W. Steeds, J. Phys. C: Solid State Phys. 18, 1 (1985).

[34] See Supplemental Material at http://link.aps.org/supplemental/ 10.1103/PhysRevB.105.064107 for Figs. S1-S4 and Table S-I containing structural information and calculations including spin-orbit coupling for $\mathrm{TaTe}_{4}$ as well as crystal and electronic structure information concerning $\mathrm{VS}_{4}$.

[35] M. D. Johannes, I. I. Mazin, and C. A. Howells, Phys. Rev. B 73, 205102 (2006).

[36] G. A. Papoian and R. Hoffmann, Angew. Chem. Int. Ed. Engl. 39, 2409 (2000).

[37] R. Patschke and M. G. Kanatzidis, Phys. Chem. Chem. Phys. 4, 3266 (2002).

[38] E. Canadell, S. Jobic, R. Brec, and J. Rouxel, J, Solid State Chem. 98, 59 (1992).

[39] J. M. Corbett, L. G. Hiltz, F. W. Boswell, J. C. Bennett, and A. Prodan, Ultramicroscopy 26, 43 (1988).

[40] M. Novak, S. Sasaki, K. Segawa, and Y. Ando, Phys. Rev. B 91, 041203(R) (2015).

[41] N. Kumar, S. N. Guin, K. Manna, C. Shekar, and C. Felser, Chem. Rev. 121, 2780 (2021). 